\title{
INTERVENÇÃO FARMACÊUTICA NO AMBIENTE HOSPITALAR
}

\section{PHARMACEUTICAL INTERVENTION IN THE HOSPITAL ENVIRONMENT}

\author{
INTERVENCIÓN FARMACÉUTICA EN EL ENTORNO HOSPITALARIO
}

\author{
Sabrina R. Wünsch ${ }^{1 *}$; Leyde Daiane de Peder ${ }^{2}$ \\ 1 - Centro Universitário Fundação Assis Gurgacz; Ceonc Hospital do Câncer \\ 2 - Professora do curso de Farmácia do Centro Universitário da Fundação Assis Gurgacz
}

\section{RESUMO:}

A farmácia hospitalar tem como objetivo principal a dispensação de medicamentos de acordo com a prescrição médica, nas quantidades e especificações solicitadas, de forma segura e no prazo requerido, promovendo o uso seguro de medicamentos e correlatos. $O$ profissional farmacêutico visa garantir a qualidade do tratamento dos pacientes, evitando erros, na hora da dispensação e na administração de medicamentos. O objetivo deste trabalho foi quantificar e identificar as intervenções farmacêuticas realizadas durante as análises de prescrições nos meses janeiro a abril de 2021 e propor melhorias para 0 processo. Trata-se de um estudo descritivo e quantitativo, onde foram analisadas 7.380 prescrições médicas. $O$ total de intervenções realizadas foi 1.156 . Durante o período estudado foram verificadas as seguintes intervenções: 705 alterações (60,98\%) na via de administração e 282 alterações $(24,39 \%)$ relacionadas a mudança de medicamentos. A não aceitação da intervenção resultando a suspenção dos medicamentos foi verificado em $14,61 \%$ (169) das intervenções. Os resultados evidenciaram a importância da triagem farmacêutica na obtenção e garantia de maior segurança e qualidade do tratamento ao paciente internado.

Palavras-chave: Assistência Farmacêutica, Farmacia, Medicamento, Paciente, Prescrições.

\section{ABSTRACT:}

The main objective of the hospital pharmacy is to dispense medication according to the medical prescription, in the quantities and specifications requested, safely and within the required period, promoting the safe use of medication and related products. The pharmacist aims to ensure the quality of treatment for patients, avoiding errors when dispensing and administering medication. The objective of this work was to quantify and identify the pharmaceutical interventions performed during the analysis of prescriptions from January to April 2021 and propose improvements to the process. This is a descriptive and quantitative study, where 7,380 medical prescriptions were analyzed, the total number of interventions made was 1,156 . Data collection was performed using a spreadsheet-like instrument, the following information was collected: the number of interventions performed that were divided into changes in the route of administration with a total of 705 changes, ie $60.98 \%$, the change of medication with 282 alterations, that is, $24.39 \%$ and, finally, the non-acceptance of the intervention resulting in the suspension of the medication with a total of 169 changes, that is, $14.61 \%$. The results showed the importance of pharmaceutical screening in obtaining a guarantee of greater safety and quality of treatment for hospitalized patients.

Descriptors: Pharmaceutical Care, Pharmacy, Medicine, Patient, Prescriptions; 


\section{RESUMEN:}

El principal objetivo de la farmacia hospitalaria es dispensar la medicación de acuerdo con la prescripción médica, en las cantidades y especificaciones solicitadas, de manera segura y dentro del plazo requerido, promoviendo el uso seguro de la medicación y productos afines. El farmacéutico tiene como objetivo garantizar la calidad del tratamiento a los pacientes, evitando errores en la dispensación y administración de la medicación. El objetivo de este trabajo fue cuantificar e identificar las intervenciones farmacéuticas realizadas durante el análisis de prescripciones de enero a abril de 2021 y proponer mejoras al proceso. Se trata de un estudio descriptivo y cuantitativo, donde se analizaron 7.380 prescripciones médicas, el total de intervenciones realizadas fue 1.156. La recogida de datos se realizó mediante un instrumento tipo hoja de cálculo, se recogió la siguiente información: el número de intervenciones realizadas que se dividieron en cambios en la vía de administración con un total de 705 cambios, es decir $60,98 \%$, el cambio de medicación con 282 alteraciones, es decir, el 24,39\% y, finalmente, la no aceptación de la intervención resultando en la suspensión de la medicación con un total de 169 cambios, es decir, el $14,61 \%$. Los resultados mostraron la importancia del cribado farmacéutico para obtener una garantía de mayor seguridad y calidad de tratamiento para los pacientes hospitalizados.

Descriptores: Asistencia Farmacéutica, Farmacia, Medicina, Paciente, Recetas;

\section{INTRODUÇÃO}

A busca constante por qualidade e melhorias nos serviços de saúde é fundamental. O profissional farmacêutico visa garantir a qualidade do tratamento dos pacientes, evitando erros, na hora da dispensação e na administração de medicamentos (SILVA, 2010). Sendo necessário a análise das prescrições por profissionais farmacêuticos capacitados.

Conforme a Resolução n 585/13 do Concelho Federal de Farmácia (CFF) objetivo do farmacêutico é de contribuir no processo de cuidado à saúde, por meio da prestação de assistência de qualidade, afim de validar a posologia, a promoção do uso racional de medicamentos, e quando há a necessidade fazer a intervenção farmacêutica. Para se prevenir possíveis erros de medicação, a equipe multidisciplinar deve relatar e documentar a aparição de situações incomuns. De modo geral, o médico é o responsável pela prescrição, o farmacêutico é pela verificação da prescrição médica, da manipulação e dispensação do medicamento e a enfermagem pela administração (CFF, 2013).

A intervenção farmacêutica ocorre quando o profissional realiza o monitoramento farmacoterapêutico, que irá identificar e resolver os problemas relacionados aos medicamentos. Devido aos diversos erros potenciais relacionados com a prescrição médica, evidencia-se a importância do farmacêutico na análise prévia à distribuição dos medicamentos, minimizando possíveis danos aos pacientes (OPAS, 2002).

Segundo o Guia de Boas Práticas em Farmácia Hospitalar e Serviço de Saúde elaborado pela Sociedade Brasileira de Farmácia Hospitalar (SBRAFH), as prescrições 
médicas devem ser analisadas pelo farmacêutico, quanto a seus componentes, quantidade, qualidade, compatibilidade, interações, possibilidade de reações adversas e estabilidade, entre outros aspectos relevantes (NOVAES, SOUZA, NÉRI et al, 2009).

As intervenções realizadas por farmacêuticos são essenciais para identificar Problemas Relacionados ao Medicamento (PRM), além disso, essa identificação proporciona medidas para a detecção e prevenção de eventos adversos (NUNES et al, 2008). Para que erros sejam evitados, a necessidade de implantar estratégias é imprescindível, assim aumentando a qualidade assistencial, diminuindo custos hospitalares, promovendo o uso racional de medicamentos, assim o que garante a segurança da terapia e consequentemente a segurança do paciente (FERRACINI, 2010).

Portanto, o presente estudo se torna importante, por meio da identificação de possíveis interações medicamentosas em prescrições de pacientes internados e posterior intervenção farmacêutica, o que pode contribuir na redução de eventos adversos, que geralmente estão associados a um maior tempo de internação, mortalidade e a maiores custos com hospitalização (CARDINAL, FERNANDES, 2014).

\section{METODOLOGIA}

A pesquisa foi realizada em um hospital oncológico situado em Cascavel, Paraná, por meio da utilização do Instrumento de Coleta de Dados e pesquisa em prescrições médicas. Foram considerados para o estudo os pacientes internados no hospital durante 0 período de janeiro a abril de 2021.

O projeto seguiu todos os preceitos éticos, iniciando após a apresentação de uma Carta de concordância do hospital oncológico, bem como, após a aprovação pelo Comitê de Ética (CEP) do Centro Universitário da FAG, sob o no 4.713 .790 em 07/05/2021 (CAAE 41090320.0000.5219).

Para a realização desta pesquisa, foram seguidos os critérios éticos descritos na Resolução Nacional de Saúde 466/12 de 12 de dezembro de 2012 e suas diretrizes e normas que regulamentam a pesquisa envolvendo seres humanos.

Cada prescrição médica passou pela triagem farmacêutica, onde foi conferido se o aprazamento das medicações está correto, bem como, a via de administração e a posologia. Verificou-se ainda se havia necessidade de intervenção. As intervenções foram realizadas quando a medicação solicitada na prescrição não era condizente à medicação padrão do hospital, dose e via de administração, nos casos de dúvidas ou divergências, o 
farmacêutico responsável pela validação das prescrições entrou em contato com o médico prescritor, para que fossem realizadas formalmente e assim serem feitas as substituições.

Todas as intervenções relatadas foram tabuladas em programa Microsoft office Excel ${ }^{\circledR} 2016$ no Centro Universitário da Fundação Assis Gurgacz.

O hospital em estudo possui capacidade de 132 leitos, sendo 6 leitos de unidade de terapia intensiva e 30 leitos do centro cirúrgico, voltados para tratamentos oncológicos. O hospital conta com 3 farmácias satélites, distribuídas nos setores do centro cirúrgico, uti e quimioterapia ambulatorial. Conta também com a farmácia central que é responsável pela distribuição dos medicamentos e insumos para todas as alas de internações.

O número de internações é de aproximadamente 1000 pacientes por mês, gerando próximo a 3000 prescrições por mês. A dispensação dos medicamentos é por dose individualizada, sendo dividida por períodos (manhã/tarde/noite) por 24 horas, que são separadas pelos técnicos de farmácia.

Evidencia-se que todas as etapas analíticas, bem como, todas as análises estatísticas descritivas, foram tabuladas através do software Microsoft office Excel ${ }^{\circledR} 2016$, os quais foram apresentados nos resultados.

\section{RESULTADOS}

Foram analisados 7.380 números de prescrições do período de janeiro de 2021 a abril de 2021 de pacientes que estiveram internados no hospital em estudo, sendo que, em situações que foram prescritos medicamentos não padronizados do hospital em estudo, houve então a necessidade de intervenções farmacêuticas.

Verificou-se que o mês com maior número de intervenções foi em janeiro, em um total de 2.130 prescrições analisadas foram realizadas intervenções em 589, isto é, $27,65 \%$. Já o mês com o menor número de intervenções foi abril, das 1.768 prescrições analisadas em 130 , isto é, em $7,35 \%$ foram necessárias intervenções.

A Figura 1 apresenta os resultados das coletas de dados a partir das prescrições que foram analisadas. 


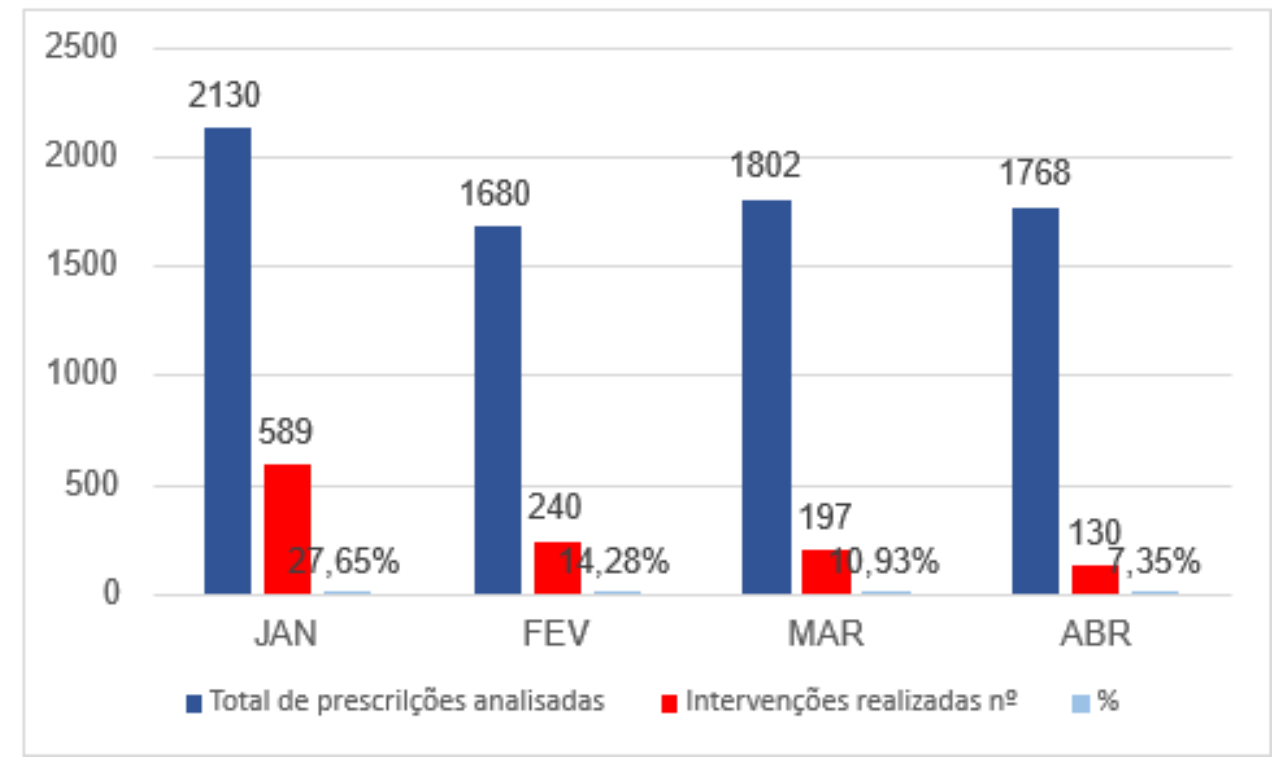

Figura 1. Número de prescrições analisadas e intervenções realizadas em um hospital de câncer situado em Cascavel, PR, 2021.

Após a coleta de dados foi possível analisar através de porcentagem a quantidade de intervenções que foram realizadas durante o período de janeiro a abril de 2021. A partir das intervenções realizadas obteve-se o número de intervenções específicas que foram feitas neste período de estudo, classificadas em mudança de via de administração da medicação, alteração dos medicamentos e intervenções que não foram aceitas, ocasionando a suspensão da medicação, como mostra a Tabela 1.

Tabela 1 - Intervenções realizadas no hospital em estudo, Cascavel, PR, 2021

\begin{tabular}{lccc}
\hline MÊS & $\begin{array}{c}\text { Mudança de via de } \\
\text { administração n (\%) }\end{array}$ & $\begin{array}{c}\text { Alteração dos } \\
\text { medicamentos n (\%) }\end{array}$ & $\begin{array}{c}\text { Medicamentos } \\
\text { suspensos n (\%) }\end{array}$ \\
\hline JAN & $423(19,85 \%)$ & $103(4,83 \%)$ & $63(2,95 \%)$ \\
FEV & $125(10,59 \%)$ & $76 \quad(4,52 \%)$ & $39(2,32 \%)$ \\
MAR & $94(5,21 \%)$ & $58(3,21 \%)$ & $45(2,49 \%)$ \\
ABR & $63(3,56 \%)$ & $45(2,54 \%)$ & $22(1,24 \%)$ \\
\hline
\end{tabular}

$\mathrm{Na}$ Tabela 2, pode-se verificar quais mudanças de via de administração foram alteradas, devido a mudança da padronização de alguns medicamentos, como o caso do 
Ondansetrona via oral (VO), passou-se a utilizar somente endovenoso (EV), assim como o Omeprazol endovenoso (EV) para a utilização do omeprazol via oral, somente em casos de não deglutição seria aplicado o EV, e em pacientes internados nas Unidades de Terapia Intensivas (UTI).

Tabela 2: Intervenções por mudança de via de administração, Cascavel, PR, 2021

\begin{tabular}{lc}
\hline Intervenção (mudança de via de administração) & $\mathbf{n}(\%)$ \\
\hline Omeprazol EV para omeprazol VO & $396(56,17 \%)$ \\
Ondansetrona VO para Ondansetrona EV & $309(43,82 \%)$ \\
\hline
\end{tabular}

As alterações de medicamentos, estão descritas na Tabela 3, assim como a quantidade de cada medicamento alterado. O grande número de alterações é devido a descontinuidade dos medicamentos, como exemplo a Heparina SC, e o Cefepime, onde houve uma dificuldade de adaptação.

Tabela 3: Intervenções realizadas na alteração de medicamentos, Cascavel, PR, 2021

\begin{tabular}{lc}
\hline Intervenção (alteração do medicamento) & n (\%) \\
\hline Heparina SC para Enoxaparina SC & $96(34,04 \%)$ \\
Cefepime para Piperacilina + Tazobactam & $61(21,63 \%)$ \\
Ranitina EV para Omeprazol VO & $58(20,56 \%)$ \\
Cefepime para Ceftazidima & $40(14,18 \%)$ \\
Cefepime para Ceftriaxona & $27(9,57 \%)$ \\
\hline
\end{tabular}

As intervenções que não foram aceitas, resultando a suspensão dos medicamentos, está demonstrada na Tabela 4, onde alguns medicamentos que foram prescritos e que não eram padrões do hospital foi suspenso ao invés de serem substituídos por medicamentos semelhantes. 
Tabela 4: Intervenções não aceitas, que levaram a suspensão do medicamento, Cascavel, PR, 2021.

\begin{tabular}{lc}
\hline Medicamentos suspensos & $\mathbf{n}(\%)$ \\
\hline Heparina SC & $97(57,39 \%)$ \\
Ranitidina EV & $20(11,83 \%)$ \\
Cimetidina EV & $19(11,24 \%)$ \\
Sulfato ferroso VO & $18(10,65 \%)$ \\
Azitromicina EV & $10(5,91 \%)$ \\
Gliconato de Cálcio VO & $5(2,95 \%)$ \\
\hline
\end{tabular}

\section{DISCUSSÃO}

A validação da prescrição médica, avaliada pelo farmacêutico responsável durante a triagem farmacêutica, é de suma importância para barrar possíveis erros, e para que não sejam levados a diante, de forma que haja a dispensação somente após aprovação do farmacêutico. As intervenções farmacêuticas efetuadas durante a validação da prescrição estão direcionadas a minimizar qualquer tipo de erro para que não aconteçam e causem danos aos pacientes. Então, durante esta triagem, quando há dúvidas ou divergências, o farmacêutico entra em contato com o médico prescritor, afim de esclarecer medicamentos ou doses, assim como medicamentos não padronizadas. Quando feitas alterações, sejam documentadas e repassas para a equipe de profissionais, para que estejam cientes, sendo feito alteração da prescrição conforme a nova adequação.

O número de prescrições analisadas neste estudo foram de 7.380 , as intervenções realizadas totalizaram 1.156, a principal intervenção está relacionada à mudança de via de administração que teve um número significativo de 705 alterações, ou seja 51\%.

A maior mudança de via de administração foi de omeprazol endovenoso (EV) para omeprazol via oral (VO), com o total de 396 alterações, ou seja $56,17 \%$. No ambiente hospitalar, os pacientes geralmente recebem medicamentos por meio de sondas nasogástricas ou enterais. Portanto, é importante que o farmacêutico considere a forma farmacêutica do medicamento utilizada nessas situações, pois o processo de dissolução e trituração de preparações orais, se não especificado, causará a destruição de 
revestimentos entéricos ou de liberação controlada, perdendo sua eficácia, podendo haver alterações na biodisponibilidade e bloqueio de sondas, ou seja, acabam obstruindo essas sondas dos pacientes, e levando a um maior risco de morte e custos de hospitalização (SANTOS, COSTA, QUEIROZ NETTO et al, 2012).

Em relação as mudanças de medicamentos, houve um número maior no mês de janeiro, que obteve 103 alterações, isto é, em 4,83\%, devido a adequação as mudanças efetivadas no hospital em estudo, sendo necessário o contato com o médico prescritor para que fossem substituídos por medicações com eficácia terapêutica semelhante do medicamento prescrito originalmente.

Em relação as alterações feitas dos medicamentos anticoagulantes, que é o caso da heparina SC que foi alterada para enoxaparina SC com o total de 96 alterações, ou seja, 34,04\%, havendo o maior número de alterações quando citado alteração de medicação. Estas alterações estão ligadas ao fato de não haver mais heparina SC no hospital em estudo. Segundo um estudo realizado sobre a anticoagulantes em pacientes oncológicos, se fez eficaz a troca desta medicação, pois a enoxaparina é uma heparina de baixo peso molecular. Pacientes com doenças neoplásicas demonstram um aumento na incidência de eventos tromboembólicos, assim associados também a terapia do câncer e aos efeitos colaterais da quimioterapia, necessitando de trombo profilaxia (RENNI et al, 2017)

Ainda falando das alterações de via de administração, obteve-se o número de 309 alterações em relação à ondansetrona via oral (VO) para ondansetrona endovenosa (EV), ou seja, com 43,82\%. Devido as intervenções em estudo terem sido realizadas em um hospital de câncer, deve-se levar em consideração que a quimioterapia ocasiona várias reações adversas, sendo uma delas a êmese, logo podemos entender as substituições feitas pelo farmacêutico responsável pela validação das prescrições (ALMEIDA; PONTES; CARDOSO, 2015).

O medicamento que ficou em segundo lugar de alterações foi o antibiótico Cefepime, que foi substituído por Piperacilina + tazobactam com o total de 61 alterações, isto é, $21,63 \%$. Segundo o Guia de Terapia Antimicrobiana (2016), nos casos de infecções pulmonares pode ser utilizado tanto as Cefalosporinas de $4^{\mathrm{a}}$ geração que é o caso do cefepime, tanto como a piperacilina + tazobactam.

As intervenções que não foram aceitas após contato com o médico prescritor somaram um total de 169 suspensões dos medicamentos durante todo o período estudado, ou seja, teve uma porcentagem de $14,61 \%$ em relação ao total de intervenções realizadas, quando comparado ao número total de intervenções que são 1.156, nos mostra que 987 
intervenções foram aceitas, ou seja, 85,38\% tiveram efetividade. Esses dados nos levam a concluir que a taxa de não aceitação dos profissionais contatados neste estudo é inferior à média encontrada em estudos com análises semelhantes, que obtiveram > 99\% de aceitação das intervenções (CARDINAL; FERNANDES, 2014).

A importância da intervenção como a qualidade do tratamento de pacientes internados, a fim de aprimorar este processo, há a necessidade de um sistema de prescrição eletrônica e prontuário eletrônicos para assim aumentarem a visualização da análise completa da prescrição pelo farmacêutico, e ferramentas de verificação de prescrição, o que nem sempre é a realidade das instituições de saúde do nosso país. 0 que dificulta a validação das prescrições é não ter evoluções detalhadas dos profissionais assistentes, falta de dados como, por exemplo, o peso para cálculo de doses de medicamentos e situações clínicas que não são evoluídas em prontuário. Recomenda-se também que haja uma equipe com treinamento para melhores resultados, desde a dispensação do medicamento até a administração do mesmo, afim de evitar quaisquer erros possíveis (CARDINAL; FERNANDES, 2014).

Após a coleta destes dados, pode-se dizer com os resultados observados, que há uma necessidade de demanda de profissionais farmacêuticos capacitados para uma análise maior em relação as prescrições, para que haja uma validação criteriosa, de acordo com os padrões estabelecidos, isto é, analisar as interações medicamentosas, a incompatibilidades de fármacos, tanto fármaco-fármaco, como fármaco-nutriente, assim como preparações medicamentosas via sonda, erros de diluições, como de administração, contribuindo com a melhora de vida do paciente, fazendo que diminua seu tempo de internamento, e o mesmo tenha uma maior adesão ao tratamento ${ }^{(12)}$.

\section{CONCLUSÃO}

No presente estudo foram realizadas 1.156 intervenções, o que correspondeu a $15,66 \%$ das prescrições analisadas. As intervenções farmacêuticas realizadas durante a validação da prescrição pelo farmacêutico responsável, mostraram-se eficazes em relação a alterações de medicamentos, assim como mudanças de via de administração, sendo diminuídas gradualmente até a adequação a nova padronização de medicamentos do hospital em estudo. Para que diminua o número de erros e consequentemente melhore a qualidade de vida dos pacientes internados, é necessário que haja sempre atenção 
farmacêutica na área hospitalar, assim, contribuindo para um menor tempo de internamento dos pacientes e uma terapia com eficácia no tratamento dos mesmos.

\section{REFERÊNCIAS}

ALMEIDA, RGL; PONTES, ACAA; CARDOSO, DA. O Manejo da Êmese em uma Unidade Oncológica: a Necessidade da Intervenção Farmacêutica em Tempo Real. Revista Brasileira de Cancerologia,Belém, v. 61, n. 2, p.115-121, 04 ago. 2015. Disponível em: <http://www.inca.gov.br/rbc/n_61/v02/pdf/05-artigo-o-manejo-da-emese-em-uma-unidadeoncologica-a-necessidade-da-intervencao-farmaceutica-em-tempo-real.pdf>. Acesso em: 02 jun. 2021

ALMEIDA R GABRIELA, A importância da validação farmacêutica da prescrição médica e o impacto na segurança do paciente em uma unidade hospitalar. (2017) Disponível em: <http://tcc.fps.edu.br:80/jspui/handle/fpsrepo/53>.

BRASIL. Conselho Federal de Farmácia. Resolução N. 585 de 29 de agosto de 2013. Regulamenta as atribuições clínicas do farmacêutico e dá outras províncias. 2013.

CARDINAL L, FERNANDES C. Intervenção Farmacêutica no processo da validação da prescrição médica. Revista Brasileira de farmácia Hospitalar 2014.

FERRACINI FT. Intervenção Farmacêutica. In Prática Farmacêutica no Ambiente Hospitalar - Do planejamento à realização. 2. ed. São Paulo: Atheneu, 2010. cap. 15, p. $185-191$.

GUIA DE TERAPIA ANTIMICROBIANA EMPÍRICA PARA SEPSE E CHOQUE SÉPTICO (2016), Disponível em <https://www.ilas.org.br/assets/arquivos/ferramentas/guia-antibioticoterapiaempirica.pdf> acesso em: 31 mai 2021.

NOVAES MRCG, SOUZA NNR, NÉRI EDR, et al. Guia de Boas Práti-cas em Farmácia Hospitalar e Serviços de Saúde. São Paulo, Ateliê Vide o Verso, 2009:11-36.

NUNES PHC, PEREIRA BMG, NOMINATO JCS, ALBUQUERQUE EM, SILVA LFN, CASTRO 
IRS, CASTILHO SR. Intervenção farmacêutica e prevenção de eventos adversos. Revista

Brasileira de Brasileira de Ciências Farmacêuticas 2008;

Organização Pan-Americana de Saúde (OPAS). Consenso Brasileiro de Atenção Farmacêutica: Proposta. Brasília: OPAS, 24 p, 2002.

RENNI MJP, CERQUEIRA MH, TRUGILHO IA, ARAUJO JUNIOR MLC, Marques MA, Koch HA. Mecanismos do tromboembolismo venoso no câncer: uma revisão da literatura. Jornal Vascular Brasileiro Vol. 16, N.4, 2017. Disponível em <https://www.scielo.br/j/jvb/a/ ZscnzKFxd4TjtP99bhTVbWg/?lang=pt\&format=pdf>acesso em: 21 mai 2021.

SANTOS CM, COSTA JM, QUEIROZ NETTO MU et al. Acompanhamento farmacoterapêutico de pacientes em uso de sonda nasoenteral em um hospital de ensino. Rev Bras Farm Hosp Serv Saúde, 2012, 3(1): 19-22

SILVA MT. Indicadores de Qualidade e Desempenho. In Ferracini FT; Borges Filho WM. Prática Farmacêutica no Ambiente Hospitalar - Do planejamento à realização. 2. ed. São Paulo: Atheneu, 2010, cap. 16, p. 193-206.

Autor para correspondência:

Sabrina R. Wünsch

Centro universitário Fag

Recebido: 04/06/2021 Aceite: 15/07/2021 